\title{
Poly-alkoxides as Functional Polymer Photocatalyst ${ }^{\dagger}$
}

\author{
Hisashi UEDA \\ National Institute of Materials and Chemical Research, \\ 1-1 Higashi, Tsukubashi, Ibaraki 305, Japan.
}

(Received January 20, 1993)

\begin{abstract}
Metal alkoxides polymerize to form metal-oxygen polymers. In the present work polymers and co-polymers of metal alkoxides were prepared and their physical and chemical properties were investigated using tetra-hydrofuran (THF) as solvent. One of the properties is photocatalytic activity and another is visible light excited ESR absorption. Tetrabutyl orthotitanate, pentaethyl niobate, and other alkoxides which dissolve in THF were polymerized under fluorescent lamps. ESR spectra were recorded while the polymer was illuminated by a halogen lamp. Catalytic activity was measured using visible light and gas chromatograph. All polymers gave visible light excited ESR spectra. The analysis of ESR intensity change caused by the light-on and light-off procedure have elucidated that there are more than two excited states, the shorter lived one of which is the reaction intermediate of photocatalysis. Among the copolymers of (- $\mathrm{TiO}-\mathrm{O}-\mathrm{NbO}-\mathrm{O}-)$ copolymers, $\mathrm{Nb} 10-20 \%$ copolymers gave the highest catalytic activity. It has also been found that the copolymer which has the largest number of $\mathrm{Ti}_{3} \mathrm{O}_{2}$ unit has the highest activity. These results seem to indicate that the catalyst polymer is a linear polymer. The merit of using alkoxide polymers and copolymers as photocatalyst lies in the facts that in these stable radical type molecules the absorption of visible light is made possible and that in these molecules the catalytic reaction sites are more accessible to the reaction substrate than 3-dimensional crystalline materials.
\end{abstract}

KEY WORDS Poly-alkoxides / Inorganic Copolymer / Photocatalyst /

Photolysis by Visible Light / Visible Light Excited ESR / Stable Free Radical

Polymer /

Little is known about 3-dimensional organic natural polymers except those foodstuff polymers as devil's tongue paste or bean-curds. On the other hands, inorganic crystals such as silicates or alumino-silicates are considered to be 3-dimensional polymers. Synthetic 3dimensional polymers are rather scarce except bakelite or cross-linked rubber.

If we use those polymers, natural or synthetic, by their macroscopic mechanical virtue, it matters little whether their structures are within the boundary of a conjectured specifications. In other words, it matters little whether the material is consisted of linear molecules or 3-dimensional molecules under the condition that the final mechanical properties satisfy our needs. Let us consider the case of photocatalytic materials. The entire reaction process may be devided into three steps. First, the catalyst must absorb light energy. Secondly, it must provide an environment in which the reaction substrate molecule is excited to a reactive state. Thirdly, the substrate molecules must reach the reaction site and must leave that site after reaction.

For all these steps, a linear molecule is superior to 3-dimensional materials because it has higher probability to meet the reaction substrate molecules than atoms in a 3dimensional materials. Therefore, a linear synthetic molecular photocatalyst is not only a synthetic substitute of a natural, 3-dimensional, and crystalline photocatalyst, but photocatalytic material of better choice.

+ Presented at the 4th SPSJ (IPC92) Conference, 1B26. 


\section{EXPERIMENTAL}

\section{Reagents}

n-Butyl orthotitanate (Tokyo Chemical Industries), Pentaethyl Niobate (Mitsubishi Metal Co.) were used as received.

\section{Polymerization Method}

The polymerization method is almost identical to the one used in the previous reports. $^{1-11)} 18.5 \mathrm{mM}$ equivalent amount of the metal alkoxide was dissolved in $500 \mathrm{ml}$ of tetrahydrofuran (THF) in a Pyrex container. To this solution $2 \mathrm{~g}$ of silica gel powder, finer than 100 mesh, were added and mixed well. The container was covered with a glass dish and placed in the center of two $30 \mathrm{~W}$ circular fluorescent lamps, so that lighting and warming of the solution occurred simultaneously. Co-polymers of metal alkoxides were obtained using a mixture of two metal alkoxides.

\section{Co-polymers of $\mathrm{Ti}$ and $\mathrm{Nb}$ alkoxides}

$\mathrm{Nb} 100 \mathrm{~mol} \%$, Ti $10 \mathrm{~mol} \%-\mathrm{Nb} 90 \mathrm{~mol} \%$, Ti $20 \mathrm{~mol} \%-\mathrm{Nb} 80 \mathrm{~mol} \%$, Ti $30 \mathrm{~mol} \%-\mathrm{Nb} 70$ mol $\%$, Ti $40 \mathrm{~mol} \%-\mathrm{Nb} 60 \mathrm{~mol} \%$, Ti $50 \mathrm{~mol} \%$ $\mathrm{Nb} 50 \mathrm{~mol} \%$, Ti $60 \mathrm{~mol} \%-\mathrm{Nb} 40 \mathrm{~mol} \%$, Ti $70 \mathrm{~mol} \%-\mathrm{Nb} \quad 30 \mathrm{~mol} \%$, Ti $80 \mathrm{~mol} \%-\mathrm{Nb}$ $20 \mathrm{~mol} \%$, Ti $90 \mathrm{~mol} \%-\mathrm{Nb} 10 \mathrm{~mol} \%$, and $\mathrm{Ti}$ $100 \mathrm{~mol} \%$ polymer samples were prepared. Their compositions were verified by fluorescent $\mathrm{X}$-rays as within $\pm 10 \%$ of the designated value in error.

\section{Measurement of Catalytic Activity}

$0.30 \mathrm{~g}$ of the polymer or a copolymer of metal alkoxides was placed in a Pyrex glass cell with two glass tube branches. The diameter of the main section of the glass cell was $20 \mathrm{~mm}$ in diameter and $150 \mathrm{~mm}$ in length. The main section was kept in the horizontal position and the side arms, in vertical position, were used as the $\mathrm{N}_{2}$ gas inlet and outflow tubes. The light source was a Toshiba UV lamp (H400P type) with yellow filters which pass light having wave lengths longer than $480 \mathrm{~nm}$. Therefore, the light of $480-700 \mathrm{~nm}$ region was actually illuminated on the catalyst. As the reaction substrate ethanol $\left(99.5 \%\right.$ with $\left.0.5 \% \mathrm{H}_{2} \mathrm{O}\right)$ was used. The reaction products, hydrogen and other volatile species, were washed by nitrogen and was analyzed by Okura 701 type gas chromatograph using Porapac-q column at $150^{\circ} \mathrm{C}$ with $\mathrm{N}_{2}$ carrier.

\section{Spectroscopic Data of the Polymers}

ESR spectra of the polymer were recorded with RE1X type spectrometer of JEOL. IR spectra were recorded by type 102 spectrometer of Japan Spectroscopic Co., Ltd. Reflectance spectra were recorded by a type- 240 spectrometer of Shimadzu Co. using a type-180/190 attachment.

\section{RESULTS}

\section{Hydrogen Generated from Ethanol by ( $\mathrm{Ti}-\mathrm{O}_{-}$}

$\mathrm{Nb}$ ) Copolymers

Figure 1 indicates the relative activities of the Ti-Nb copolymers when used as photocatalysts to decompose ethanol with visible light. Catalytic activity was the highest when $10-20 \mathrm{~mol} \%$ of $\mathrm{Nb}$ existed in the copolymer.

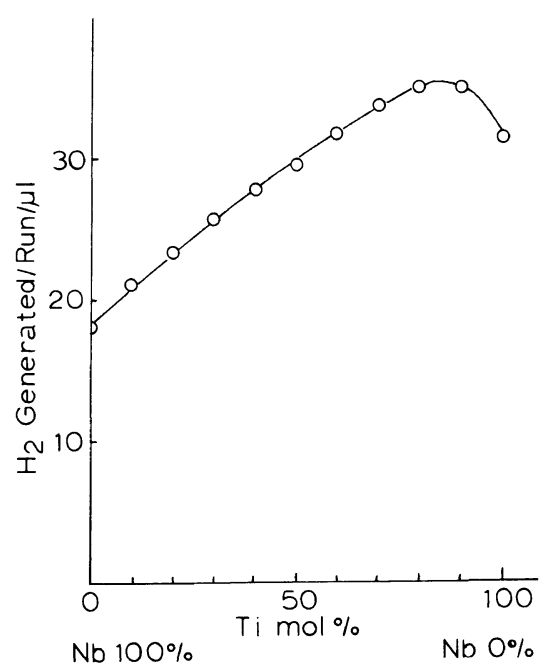

Figure 1. Photocatalytic activities of $(-\mathrm{Ti}-\mathrm{O}-\mathrm{Nb}-)$ copolymers when used for photolysis of ethanol. 


\section{Reflectance Spectra of Polymers}

Figure 2 indicates the reflectance spectra of the polymers thus obtained. These spectra show that the relatively stronger visible light absorption region of $\mathrm{Ti}-\mathrm{O}-\mathrm{Nb}$ copolymers is more extended toward $600-800 \mathrm{~nm}$ than either Ti $100 \%$ polymer or $\mathrm{Nb} 100 \%$ polymer.

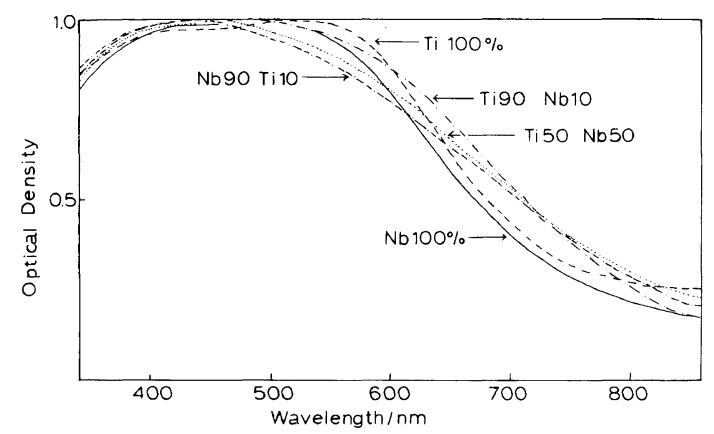

Figure 2. Reflectance spectra of $(-\mathrm{Ti}-\mathrm{O}-\mathrm{Nb}-)$ copolymers.

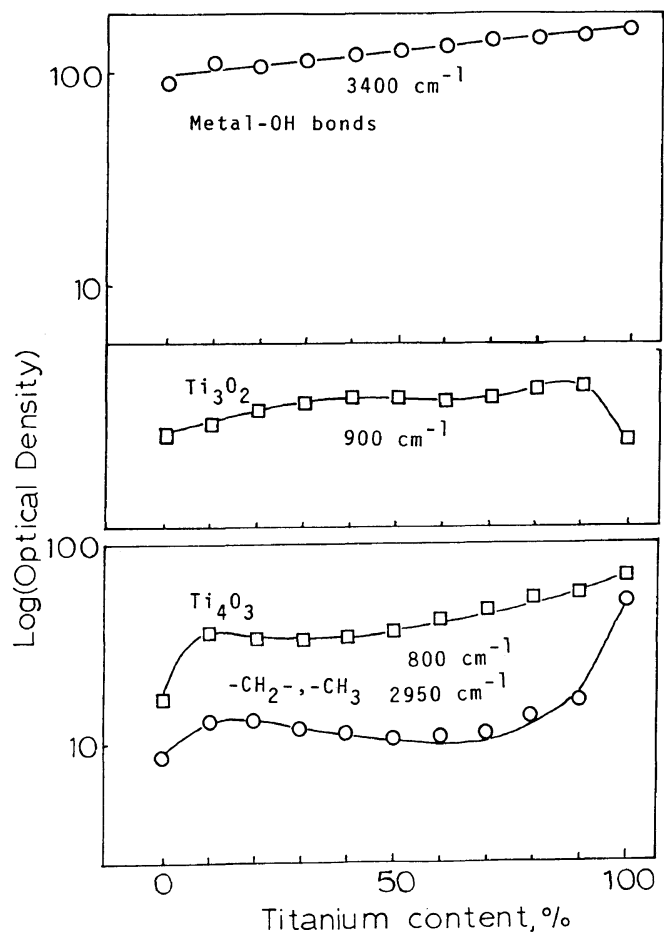

Figure 3. IR spectra of $(-\mathrm{Ti}-\mathrm{O}-\mathrm{Nb}-)$ copolymers.

\section{Infrared Spectra of Polymers}

Figure 3 shows the IR spectra of $\mathrm{Ti}-\mathrm{O}-\mathrm{Nb}$ copolymers. The metal-OH bonds linearly increase with the $\mathrm{Ti}$ atom increase. $\mathrm{Ti}_{3} \mathrm{O}_{2}$ units sharply decreases when $\mathrm{Ti}$ content reaches $100 \%$. The $-\mathrm{CH}_{2}-$ and $-\mathrm{CH}_{3}$ groups due to butyl or ethyl groups increase when Ti content is above $90 \%$. Therefore, free radical content $\left({ }^{-} \mathrm{Ti}-\mathrm{OBu}(=\mathrm{O})\right)$ is large when $\mathrm{Ti}$ content is almost $100 \%$. Thus, the number of surface $\mathrm{OH}$ groups is proportional to the Ti content. In a $50: 50$ molar mixture $(\mathrm{Ti}: \mathrm{Nb})$ of $(\mathrm{Ti}-\mathrm{O}-\mathrm{Nb})$ copolymer, $\mathrm{Ti}_{3} \mathrm{O}_{2}$ and $\mathrm{Nb}_{3} \mathrm{O}_{2}$ units still exist to a considerable extent.

\section{ESR Spectra of the Copolymers}

Figure 4 shows the ESR spectra of $\mathrm{Ti}-\mathrm{O}-\mathrm{Nb}$ copolymers when no light is illuminated on them. These spectra indicate that these polymers are stable free radicals. The differences in the shapes of the spectra indicate that

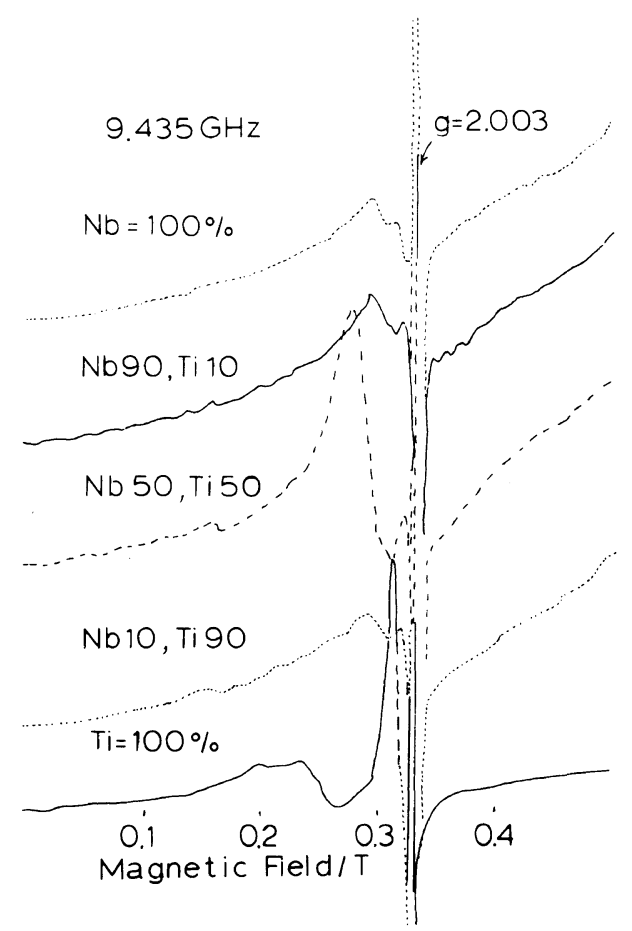

Figure 4. ESR spectra of $(-\mathrm{Ti}-\mathrm{O}-\mathrm{Nb}-)$ copolymers when light is not illuminated. 


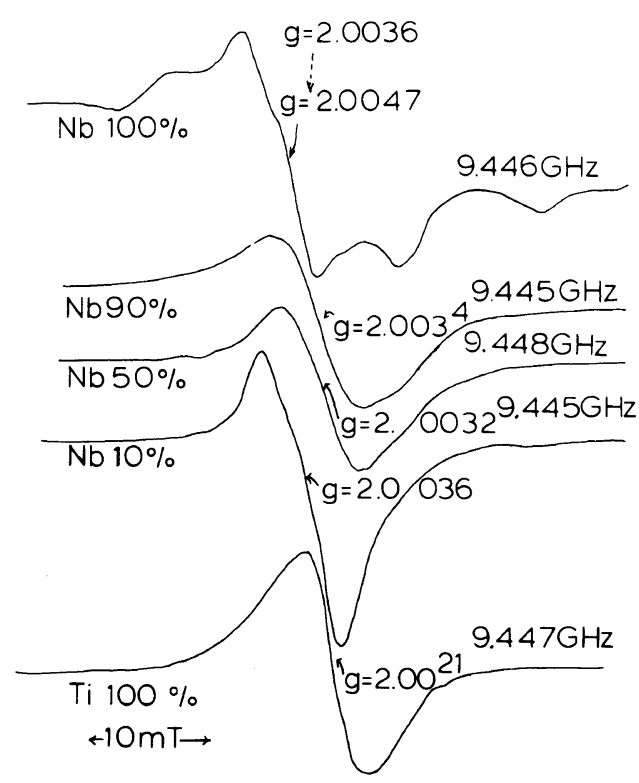

Figure 5. ESR spectra of $(-\mathrm{Ti}-\mathrm{O}-\mathrm{Nb}-)$ copolymers when illuminated by visible light.

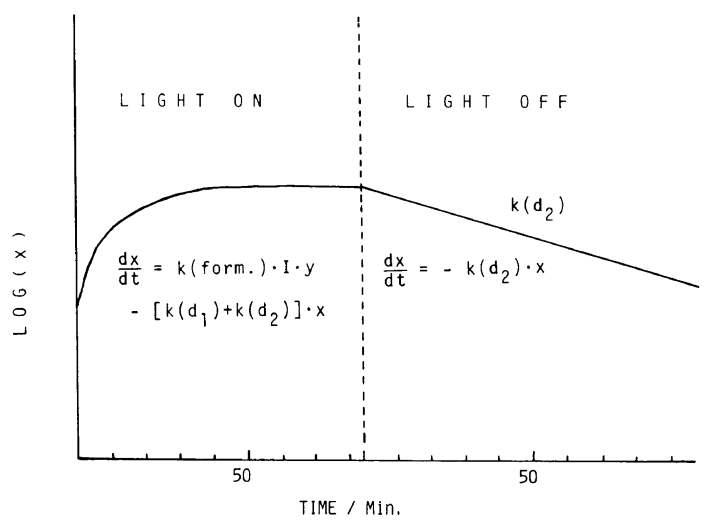

Figure 6. ESR intensity change with time, with and without light.

the unpaired electron has spin density on several metal atoms. Figure 5 shows the ESR spectra of these polymers when visible light is illuminated.

ESR Intensity Change during and after Visible Light Illumination

Figure 6 shows ESR intensity change (the intensity across $g=2.0021$ of the spectrum for
Table I. Decay rate constants of excited species

\begin{tabular}{cccc}
\hline $\mathrm{Ti}: \mathrm{Nb}$ & $k_{11} / 10^{-2}$ & $k_{12} / 10^{-2}$ & $k_{2} / 10^{-3}$ \\
\hline $100: 0$ & 4.969 & 4.969 & 1.799 \\
$90: 10$ & 5.768 & 5.768 & 3.200 \\
$50: 50$ & 7.730 & 3.896 & 2.833 \\
$10: 90$ & 14.65 & 6.244 & 3.293 \\
$0: 100$ & 10.86 & 3.259 & 4.894 \\
\hline
\end{tabular}

$\mathrm{Ti}=100 \%$ case in Figure 5 , the shape of the spectrum did not change during formation and decay), versus time, when the visible light is illuminated and turned off. When the light is turned on, the ESR signal increases until total intensity comes to a saturation value. After attaining this saturation value, if the light is turned off, the intensity decreases at a semilogarithmic-linear rate. The decay rate constants observed for five different samples of ( $\mathrm{Ti}-\mathrm{O}-\mathrm{Nb}$ ) copolymers are listed in Table I. In Table I, $k_{11}$ and $k_{12}$ are two components of $k\left(d_{1}\right), k_{11}$ being the first section and $k_{12}$ being the second section of a growing curve as shown in Figure 6. $k_{2}$ is identical to $k\left(d_{2}\right)$ in Figure 6 . The decay rate constant in the growing section of light-on experiment has two components, $k_{11}$ and $k_{12}$, if the $\mathrm{Nb}$ content is $50 \%, 90 \%$, and $100 \%$. It seems that these two decay components are related to $\mathrm{Nb}$ atom.

\section{DISCUSSION}

\section{Structure of the Obtained Polymer}

Polymers of metal alkoxides obtained by partial-hydrolysis-polymerization have been described by Bradley. ${ }^{1,2}$ According to him, there are five directions in which polymerization reactions proceed. ${ }^{3}$ Those partial-hydrolysis-methods employed water as a component of the solvent. In the present work, dry THF was used and, therefore, the polymerization reaction seemed to proceed only in one direction, in which the polymerization took place most easily. As a result, the rate of polymerization is relatively slow compared to 
-(TiO-O-TiOBu-O)n- Polymer

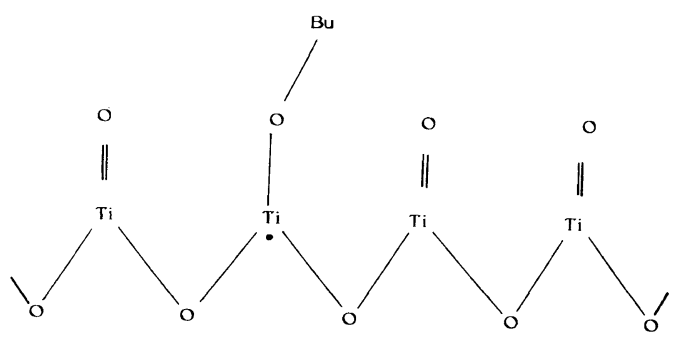

$-(\mathrm{TiO}-\mathrm{O}-\mathrm{NbO}-\mathrm{O})_{\mathrm{n}}-$ Copolymer

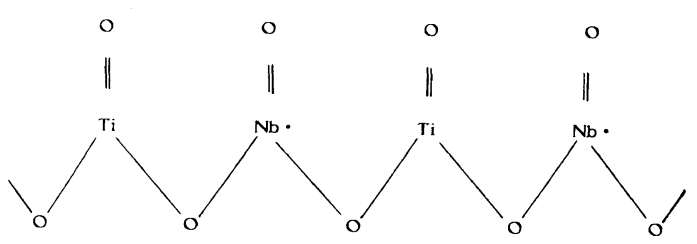

Figure 7. The chemical structures of alkoxide polymers. Top, titanium $100 \%$ polymer; Bottom, $\mathrm{Ti} /(\mathrm{Ti}+\mathrm{Nb})=50 \%$ polymer.

hydrolysis cases. Evidence to prove this one directional polymerization has been given by X-ray diffraction and IR absorption spectra. ${ }^{4}$ By X-ray data, the polymer obtained was found to be an amorphous material. Elementary analysis results indicated that the composition of the polymer obtained has the molecular formula approximately that of $\mathrm{TiO}_{2}$ but with considerable amounts of carbon and hydrogen. The presence of a considerable amount of $\mathrm{Ti}_{3} \mathrm{O}_{2}$ units in the polymer molecule was concluded from IR spectra. ${ }^{4}$ These data will allow to draw a structural picture as shown in Figure 7.

\section{Reaction Diagram}

The light excited intensity of ESR absorption, $x$, changes as shown in Figure 6. The curves in Figure 6 consists of three component curves, (a), (b), and (c). (a) is the growing curve which has $k$ (formation), (b) is the decay curve

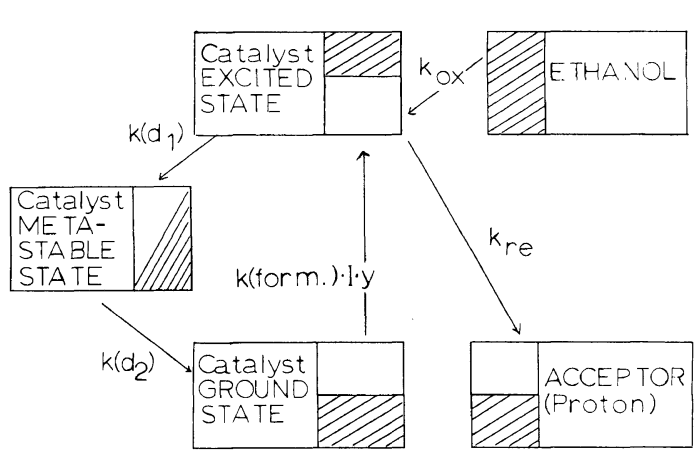

Figure 8. Block diagram of the excitation and reaction scheme of catalysis reactions.

which appears only during light illumination and has a decay constant $k\left(d_{1}\right)$. (c) is the decay curve which appears during light-on and light-off periods and has a decay constant $k\left(d_{2}\right)$. Therefore,

$$
\mathrm{d} x / \mathrm{d} t=k(\text { formation }) \cdot I \cdot y-\left\{k\left(d_{1}\right)+k\left(d_{2}\right)\right\} \cdot x
$$

$\mathrm{d} x / \mathrm{d} t=-k\left(d_{2}\right) x$

(1) is for a light-on case and (2) is for a light-off case. $I$ is the visible light intensity illuminated on the catalyst. $y$ is the concentration of the ground state catalyst molecule, $Y$. The light excited catalyst molecule $X$ has two subspecies, $X_{1}$ and $X_{2} . X_{1}$ is a short lived species and $X_{2}$ is a long lived species and can be observed even after light is turned off. The decay constant of $X_{1}$ is $k\left(d_{1}\right)$ and the decay constant of $X_{2}$ is $k\left(d_{2}\right) . X_{2}$ corresponds to the metastable state in Figure 8 . Since $k\left(d_{1}\right) \gg$ $k\left(d_{2}\right),(1)$ may be approximated by $\left(1^{\prime}\right)$,

$$
\mathrm{d} x / \mathrm{d} t=k(\text { formation }) \cdot I \cdot y-k\left(d_{1}\right) \cdot x
$$

By integration,

$$
\begin{array}{r}
x=x_{\infty}\left[1-\exp \left\{-k\left(d_{1}\right)-k\left(d_{2}\right)\right\}\right] \\
\text { light on case, } \\
x=x_{\infty} \cdot \exp \left[-k\left(d_{2}\right) t\right] \\
\text { light off case, }
\end{array}
$$

are obtained, $x_{\infty}$ being $\left\{k_{\mathrm{f}} /\left(k_{1}+k_{2}\right)\right\} \cdot I \cdot y$. From the curves in Figure 6 , these rate 
constants are determined, and shown in Table I. In the table, $k\left(d_{1}\right)=k_{11}$ and $k_{12}, k\left(d_{2}\right)=k_{2}$. From these results, it is possible to draw a reaction scheme as shown in Figure 8. In Figure 8 , the ground state of the catalyst molecule $Y$ is excited to the excited state of the catalyst molecule $X_{1}$ by visible light. Therefore,

$$
\begin{aligned}
& \quad \stackrel{\text { light }}{\longrightarrow} X_{1} \\
& X_{1}+\mathrm{C}_{2} \mathrm{H}_{5} \mathrm{OH} \longrightarrow Y^{-}+\mathrm{C}_{2} \mathrm{H}_{5} \mathrm{OH}^{+} \\
& Y^{-}+\mathrm{H}^{+} \quad \longrightarrow .5 \mathrm{H}_{2}+Y \\
& \mathrm{C}_{2} \mathrm{H}_{5} \mathrm{OH}^{+} \quad \longrightarrow \text { Oxidation Product }+\mathrm{H}^{+} \\
& X_{1} \longrightarrow X_{2} \longrightarrow Y
\end{aligned}
$$

In Figure 8, the shadowed portions in the block diagram vaguely denote the energy states of the electrons: the ground state, metastable state, and excited state.

The original structure of $-\mathrm{Ti}\left(\mathrm{OC}_{4} \mathrm{H}_{9}\right)_{2}$ - is a tetrahedral configuration as shown in Figure 9A. An energy level diagram as shown in Figure $9 \mathrm{~B}$ is drawn upon this structure, if one electron exists in the $3 \mathrm{~d}$ orbital of Tiatom. Therefore, if one $\mathrm{OC}_{4} \mathrm{H}_{9}$ groups is eliminated, the $d^{2}$ orbitals, $x^{2}-y^{2}$ and $z^{2}$, will be used to form hybrid orbitals $d^{2}$ s sigma bonds. Then the extra Ti electron will occupy one of the $4 p$ orbitals. Dark ESR absorption corresponds to this $4 p^{1}$ (Ti) electron (Figure 9C). When visible light is illuminated, one of the $d^{2}$ s electrons is excited to $4 p$ orbital to make a $4 p^{2}$ (Ti) structure and the resulting unpaired spin density is mainly in $4 \mathrm{~s}$ orbital of Ti. The narrow line width of the light-excited ESR spectra in Figure 5 is explained by this structure. The $-\mathrm{CH}_{2}-\mathrm{CH}_{3}$ hyperfine structure observed in the light excited ESR spectrum of Ti-alkoxide polymer ${ }^{4}$ may be explained well by this $4 \mathrm{~s}$-spin density model, because $=\mathrm{Ti}-\mathrm{O}-\mathrm{C}-\mathrm{C}-\mathrm{C}-\mathrm{CH}_{3}$ seems to form a hexagonal ring structure in which $(\mathrm{Ti}, 4 \mathrm{~s})$ orbital and $\left(\mathrm{CH}_{3}, \mathrm{C}-\mathrm{H}, \sigma\right)$ orbitals overlap each other.

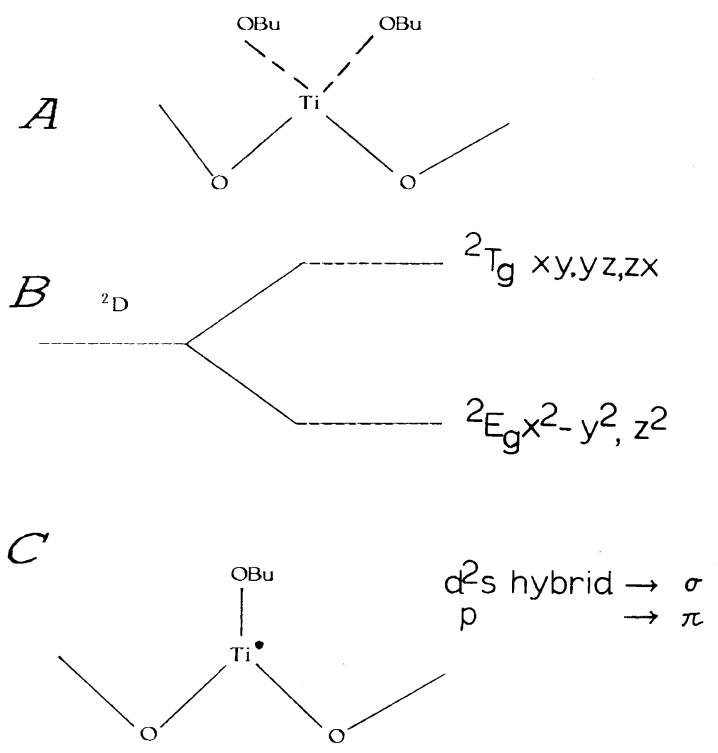

Figure 9. Configuration of titanium atom. A: Two $\mathrm{OC}_{4} \mathrm{H}_{9}$ groups not eliminated by condensation. B: Electronic state of $\mathrm{Ti} 3 \mathrm{~d}^{\mathbf{1}}$, assuming tetrahedral environment. C: Structure when one $\mathrm{OC}_{4} \mathrm{H}_{9}$ group is taken away.

\section{Mechanism of Visible Light Absorption}

Figure 10 illustrates the visible light absorption of alkoxide polymer catalyst. In case of an ordinary diamagnetic type crystalline catalyst, the light absorption leads to an excitation of a valence band electron to the conduction band. In the case of stable paramagnetic molecules, the ground state has $M_{\mathrm{s}}=1 / 2$ spin state and therefore there are several spin-excited states having similar energy. The actual ground state is, therefore, a hybrid of these spin-excited states and so the ground state energy level goes up compared to the diamagnetic case. The light-excited state is also composed of a hybrid of spin-excited states having $\left(M_{\mathrm{s}}=1 / 2\right.$ and $\left.M_{s}=3 / 2\right)$, whose lowest orbital component has considerably lower energy than diamagnetic case. As a result, the energy difference between a ground state orbital and excited state orbital is far smaller than the diamagnetic case. Thus, light with a wavelength $480-700 \mathrm{~nm}$ can be used to photolyse ethanol.

In a continuous solid, it is convenient to 


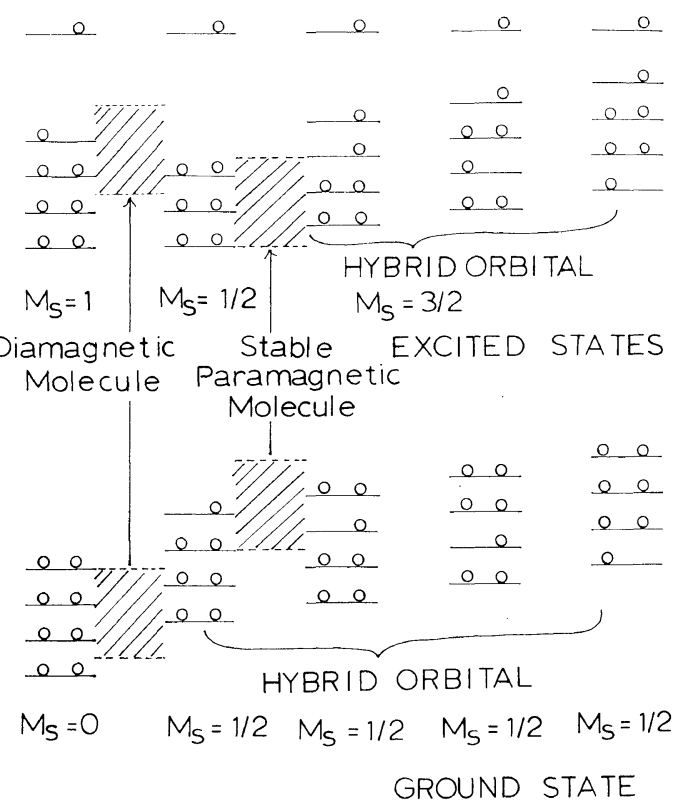

Figure 10. Molecular orbital energy levels (ground and photoexcited states) of diamagnetic and paramagnetic inorganic polymer molecules.

describe the behavior and energy state of electrons by the band theory. The band theory is based on the idea that all electrons contained in the solid are exchangeable. In the case of a low-dimensional solid, the extent of the exchangeable region is somehow narrowed. For this reason, a molecular orbital approach seems to be better to analyse a linear molecule photocatalyst system.

\section{Special Functions of Inorganic Polymers and Copolymers}

The most characteristic point of an inorganic polymer is it can be a stable free radical polymer and a linear polymer. If these two conditions are simultaneously satisfied, they make the polymer catalyst work in the visible wavelength region. Stable free radical, $M_{s}=1 / 2$ state, makes the energy difference between the ground state and the excited state small. This resembles the case of impurity doped semiconductor in which the energy band is bent around an impurity ion. The linear polymer makes the reaction substrate more accessible to the charge transfer site at which electric charge with higher electrostatic energy is transferred from the catalyst to the reaction substrate molecule. In the case of a three dimentional crystalline catalyst, the rigid crystalline structure prevents the penetration of the substrate into catalyst body and therefore only the crystal surfaces can be used. Therefore, the efficiency is low.

Such an advantage of a linear structure is more significant in the case of co-polymers. In a $(-\mathrm{NbO}-\mathrm{O}-\mathrm{TiO}-)$ copolymer with 10 $20 \% \mathrm{Nb} /(\mathrm{Nb}+\mathrm{Ti})$ and $90-80 \% \mathrm{Ti} /(\mathrm{Nb}+$ $\mathrm{Ti}$ ), the catalyst has a substructure like -O$\left(\mathrm{TiO}_{2}\right)_{9-x}\left(\mathrm{TiO}_{2} \mathrm{R}\right)_{x}-\mathrm{NbO}-\mathrm{O}-$ or like -O $\mathrm{NbO}-\mathrm{O}-\left(\mathrm{TiO}_{2}\right)_{8-x}\left(\mathrm{TiO}_{2} \mathrm{R}\right)_{x}-\mathrm{NbO}-\mathrm{O}-$, which works as an effective catalytic unit. This, it seems, establishes a molecular orbital with member atoms limited to one of the substructures. An electron, light-excited to an unoccupied molecular orbital having relatively small member atoms, will have a higher flatband potential. ${ }^{12}$ For these reasons, a study of inorganic copolymers or inorganic-organic copolymers of metal alkoxide give important data when these polymers are chosen for photocatalysts.

\section{REFERENCES}

1. D. C. Bradley, R. Gaze, and W. Wardlaw, J. Chem. Soc., 721, 3977 (1955); 469 (1957).

2. D. C. Bradley and H. Holloway, Can. J. Chem., 40, 1176 (1962).

3. D. C. Bradley, Coord. Chem. Rev., 2, 299 (1967).

4. T. Sekine, M. Yonemura, and H. Ueda, Nippon Kagaku Kaishi, 605 (1985).

5. M. Yonemura, T. Sekine, and H. Ueda, Chem. Lett., 487 (1986).

6. H. Ueda, M. Yonemura, and T. Sekine, J. Mater. Sci., 22, 3349 (1987).

7. H. Ueda, J. Polym. Sci., A, 27, 263 (1989).

8. H. Ueda, Nippon Kagaku Kaishi, 1194 (1988).

9. H. Ueda, Nippon Kagaku Kaishi, 202 (1990).

10. H. Ueda, Denki Kagaku, 57, 1141 (1989).

11. H. Ueda, J. Materials Sci. Lett., 9, 203 (1990).

12. H. Tsubomura, "Photoelectrochemistry and Energy Conversion," Tokyo Kagakudoujin, Tokyo, 1981, p 155. 